\title{
Comprehensive measurement of pp-chain solar neutrinos with Borexino
}

\author{
Mariia Redchuk* \\ Institut für Kernphysik, Forschungszentrum Jülich, Jülich, Germany \\ III. Physikalisches Institut B, RWTH Aachen University, Aachen, Germany \\ E-mail: m.redchukefz-juelich.de
}

\begin{abstract}
* on behalf of the Borexino Collaboration:
M. Agostini, K. Altenmüller, S. Appel, V. Atroshchenko, Z. Bagdasarian, D. Basilico, G. Bellini, J. Benziger, D. Bick, G. Bonfini, D. Bravo, B. Caccianiga, F. Calaprice, A. Caminata, L. Cappelli, P. Cavalcante, F. Cavanna, A. Chepurnov, K. Choi, D. D’Angelo, S. Davini, A. Derbin, A. Di Giacinto, V. Di Marcello, X.F. Ding, A. Di Ludovico, L. Di Noto, I. Drachnev, K. Fomenko, A. Formozov, D. Franco, F. Gabriele, C. Galbiati, M. Gschwender, C. Ghiano, M. Giammarchi, A. Goretti, M. Gromov, D. Guffanti, C. Hagner, E. Hungerford, Aldo Ianni, Andrea Ianni, A. Jany, D. Jeschke, V. Kobychev, G. Korga, S. Kumaran, T. Lachenmaier, M. Laubenstein, E. Litvinovich, P. Lombardi, I. Lomskaya, L. Ludhova, G. Lukyanchenko, L. Lukyanchenko, I. Machulin, G. Manuzio, S. Marcocci, J. Maricic, J. Martyn, E. Meroni, M. Meyer, L. Miramonti, M. Misiaszek, V. Muratova, B. Neumair, M. Nieslony, L. Oberauer, V. Orekhov, F. Ortica, M. Pallavicini, L. Papp, Ö. Penek, L. Pietrofaccia, N. Pilipenko, A. Pocar, G. Raikov, M.T. Ranalli, G. Ranucci, A. Razeto, A. Re, M.Redchuk, A. Romani, N. Rossi, S. Rottenanger, S. Schönert, D. Semenov, M. Skorokhvatov, O. Smirnov, A. Sotnikov, Y. Suvorov, R. Tartaglia, G. Testera, J. Thurn, E. Unzhakov, A. Vishneva, R.B. Vogelaar, F. von Feilitzsch, M. Wojcik, M. Wurm, O. Zaimidoroga, S. Zavatarelli, K. Zuber.
\end{abstract}

A comprehensive measurement of solar neutrino fluxes has been completed using the Borexino PhaseII data in an extended energy range. The measurement reports $p p,{ }^{7} \mathrm{Be}$, and pep neutrino fluxes with the highest precision ever achieved, ${ }^{8} \mathrm{~B}$ with the lowest energy threshold, the first Borexino limit on hep neutrinos, as well as the best limit on CNO neutrinos. These results and their physics interpretations concerning the so-called solar metallicity puzzle and the electron-neutrino survival probability, as well as other highlights of the analysis, have been summarized in this talk. 


\section{Solar neutrinos}

The Sun is powered by nuclear fusion reactions that convert hydrogen into helium. More than $99 \%$ of the solar energy originates in the so-called $p p$-chain reaction. An alternative $\mathrm{CNO}$ catalytic cycle is hypothesized to contribute up to $1 \%$ of the energy output, but it has never been observed yet. Both processes are depicted in Fig. 1, and different types of neutrinos originating at different stages of the reactions are highlighted. Neutrinos that are the products of these reactions are the only direct carriers of information about the processes in the core of the Sun.

pp chain

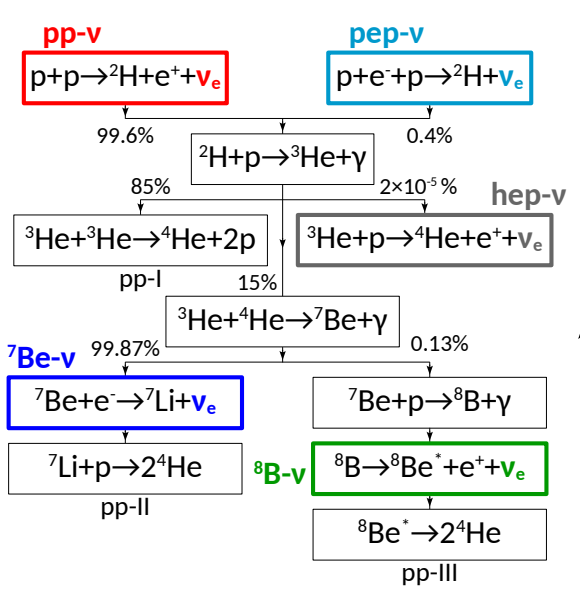

CNO cycle

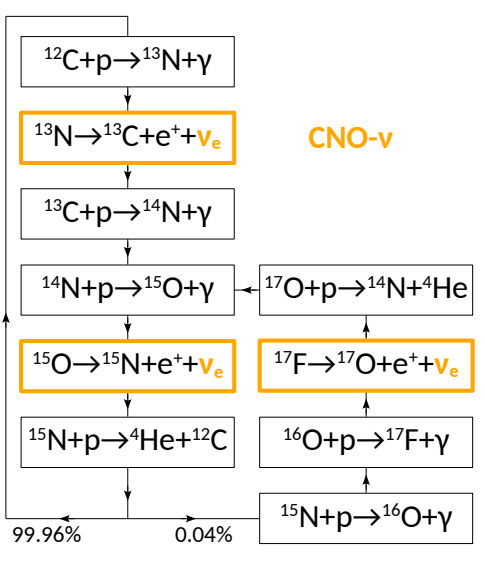

Figure 1: The $p p$ chain and the $\mathrm{CNO}$ cycle reactions producing the so-called $p p, p e p,{ }^{7} \mathrm{Be},{ }^{8} \mathrm{~B}$, hep, and $\mathrm{CNO}$ neutrinos.

While the spectral shapes are defined by the fusion reactions, the total flux depends on many parameters that vary according to the assumed composition of the Sun, in particular the fraction of the heavy elements. Standard Solar Models (SSMs) predict the values of the total fluxes based on different compositions, and are divided into two classes, low and high metallicity (LM and HM, respectively), depending on the fraction of elements heavier than helium. An example of solar neutrino spectra resulting from one type of LM-SSM called B16 [1] are shown in Fig. 2a.

Current knowledge about these models does not provide enough information to determine the metallicity of the Sun. By measuring the solar neutrino fluxes we are able to study the reactions in the $p p$ chain. Furthermore, using the knowledge about the fluxes and assuming a certain solar model, we probe the neutrino oscillations theory for deviations from standard interactions; while adopting the MSW-LMA theory of neutrino oscillations [2] we test SSMs. Apart from the pp-chain neutrinos, the measurement of the flux of CNO neutrinos would help us solve the metallicity puzzle described above.

\section{The Borexino detector}

Borexino is a liquid scintillator detector the primary goal of which is measuring the fluxes of solar neutrinos [3]. It is located in the Laboratori Nazionali del Gran Sasso in the mountains of Italy at $3800 \mathrm{~m}$ water-equivalent depth. In 2012 Borexino started Phase-II of data taking which is characterized by its higher radiopurity. This was made possible due to extensive purification campaigns in 2010 and 2011 after which the already unprecedentedly low radioactive background of the detector was improved even more. The schematic representation of the detector is shown in Fig. 2b. It is nominally equipped with 2200 photomultiplier tubes (PMTs) mounted on the Stainless Steel Sphere (SSS) that point inwards into the Inner Detector (ID), which contains the scintillator; and 220 PMTs pointing outwards in the Outer Detector which serves the purpose of a Cherenkov muon veto and a shield against 
external background. Apart from that, the ID contains two thin nylon spheres which protect the scintillator from radon diffusion from the outside. [4].

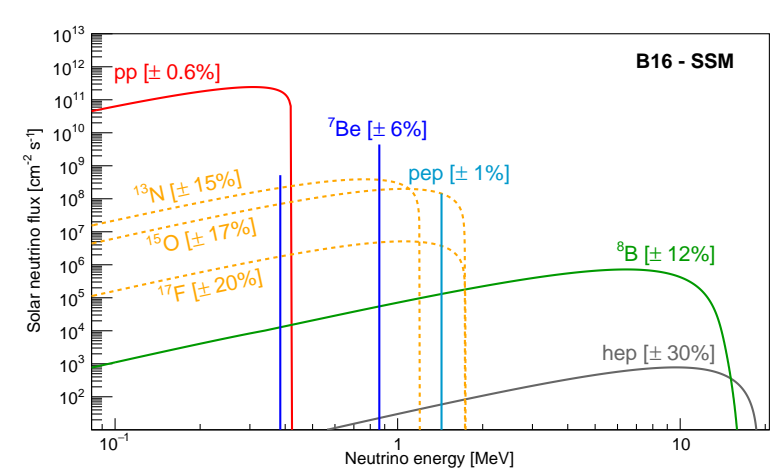

(a)

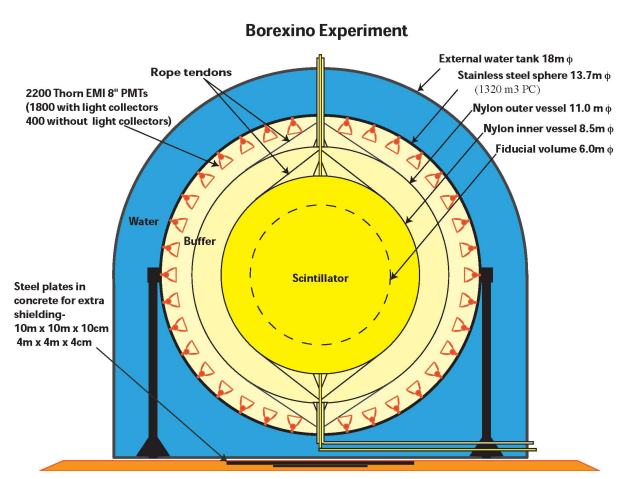

(b)

Figure 2: (a) The spectra of the solar neutrinos in units of $\mathrm{cm}^{-2} \mathrm{~s}^{-1} \mathrm{MeV}^{-1}$ for continuous spectra and $\mathrm{cm}^{-2} \mathrm{~s}^{-1}$ for monoenergetic lines. (b) A schematic representation of the Borexino detector.

\section{The Borexino solar neutrino analysis}

The solar neutrino analysis is divided into two parts with different approaches: the Low Energy Region (LER, Sec. 3.1) and High Energy Region (HER, Sec. 3.2). The LER analysis results in the measurement of the rates of $p p$,

${ }^{7} \mathrm{Be}$, and pep neutrinos, as well as an upper limit on CNO neutrinos; while ${ }^{8} \mathrm{~B}$ neutrino measurement is conducted in the HER analysis, with an additional study on the rare hep neutrinos. In both HER and LER, the steps to obtain the final neutrino rates are similar. The first step is to obtain a spectrum of neutrino-like events using selection cuts designed to remove unwanted events. Then, in order to extract the neutrino rates, fits of global variables are performed.

\subsection{Low energy region analysis}

\subsubsection{Data selection}

The data used in this analysis covers the so-called Phase-II of the Borexino dataset which spans from December 2011 to May 2016 and covers the exposure of 1291.51 days $\times 71.3$ tons. Some of the prominent selection cuts are the following:

- Muon cut: removes muon events. The efficiency of this cut is $\sim 99.992 \%$.

- Muon daughter cut: removes the products of muon spallation in the LS and effects of electronics saturation, via applying a $300 \mathrm{~ms}$ veto after each muon event.

- Fiducial Volume cut: removes events far from the center of the detector in order to maximize the active volume while minimizing radioactive background coming from the nylon spheres, PMTs, and the SSS.

Apart from these cuts, a special Three-fold coincidence (TFC) method is applied, which does not remove any events from the sample, but rather divides it into two categories, rich and depleted in ${ }^{11} \mathrm{C}$. Cosmogenic ${ }^{11} \mathrm{C}$ background originates from muon spallation on the ${ }^{11} \mathrm{C}$ molecules present in the LS:

$$
\mu+{ }^{12} \mathrm{C} \rightarrow \mu+{ }^{11} \mathrm{C}+n .
$$

The TFC algorithm looks for coincidences of a muon event and a neutron capture $(n+p \rightarrow D+\gamma)$ which happens on average $\sim 250 \mu$ s after the muon. After such a coincidence is found, a cylindrical volume following the muon path is constructed, as demonstrated in Fig. 3a. All the events in each cylinder are taken to contribute to 
the so-called TFC-tagged spectrum containing $\sim 92 \%$ of ${ }^{11} \mathrm{C}$ and $\sim 40 \%$ of exposure; and the remaining $60 \%$ of the exposure constitutes the TFC-subtracted spectrum with the rest of the ${ }^{11} \mathrm{C}$ events. Both spectra are used in the multivariate fit. The effect of the TFC subtraction as well as the cuts described before can be seen in Fig. 3b.

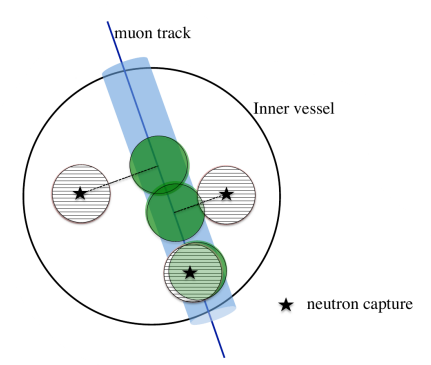

(a)

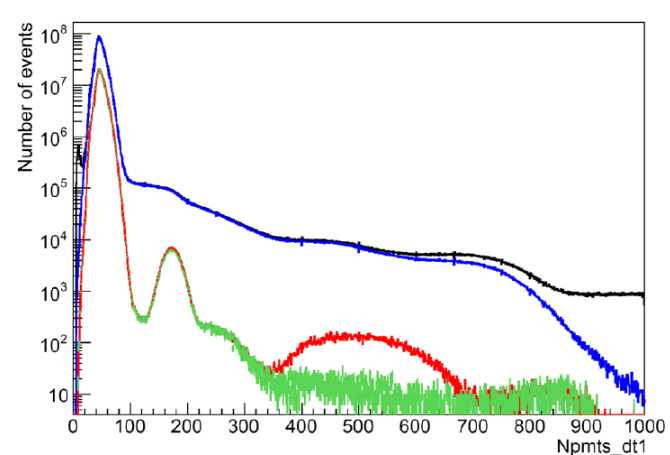

(b)

Figure 3: (a) Schematic representation of a muon track passing through the detector (blue line) and the region around it where high amounts of ${ }^{11} \mathrm{C}$ are expected to be found (light blue cylinder). (b) Phase-II energy spectrum of all Borexino events (black) and the spectra after applying the muon and muon daughter cut (blue), FV cut (red), and TFC cut (green) in this order.

\subsubsection{Multivariate fit}

The principle of the multivariate fit is to use a product of multiple likelihood functions that depend on the same parameters. In the Borexino LER analysis, four spectra contribute to the multivariate likelihood:

$$
\mathscr{L}_{\mathrm{MV}}(\vec{\theta})=\mathscr{L}_{\mathrm{TFC} \text {-tagged }}(\vec{\theta}) \cdot \mathscr{L}_{\mathrm{TFC}-\mathrm{sub}}(\vec{\theta}) \cdot \mathscr{L}_{\mathrm{RD}}(\vec{\theta}) \cdot \mathscr{L}_{\mathrm{PS}}(\vec{\theta}),
$$

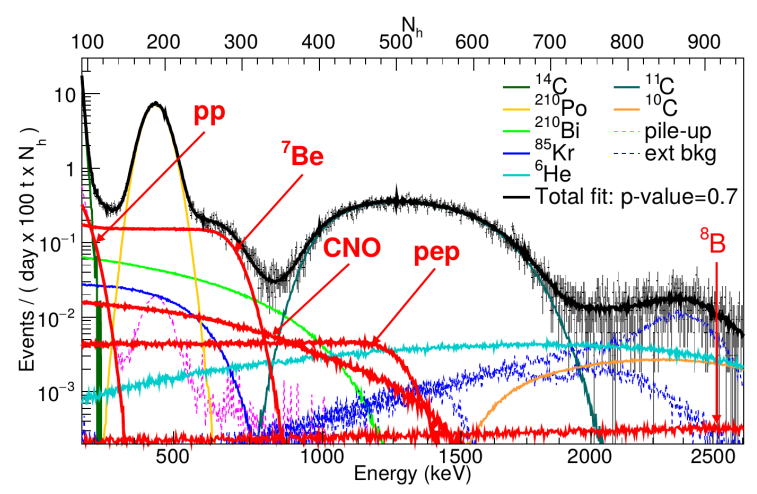

(a) The TFC-tagged energy spectrum.

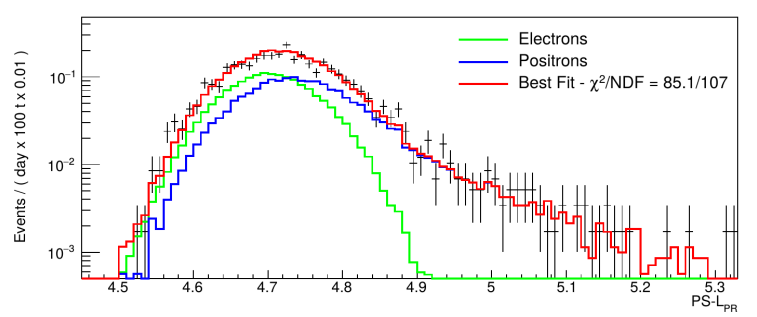

(c) Pulse shape variable distribution of the TFCsubtracted data sample.

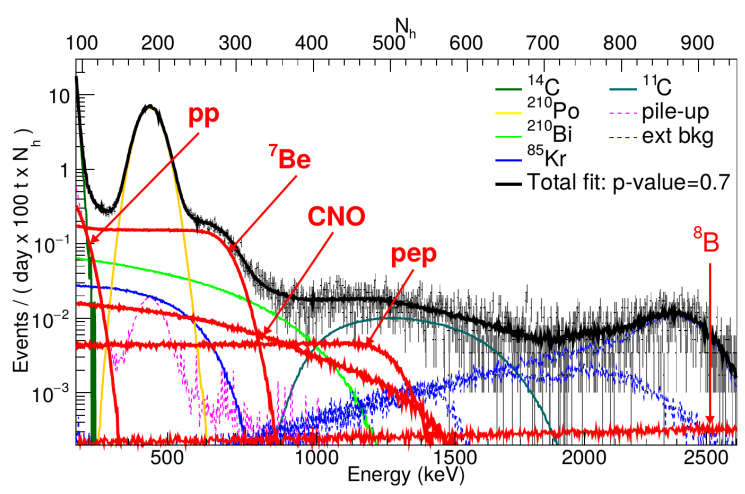

(b) The TFC-subtracted energy spectrum.

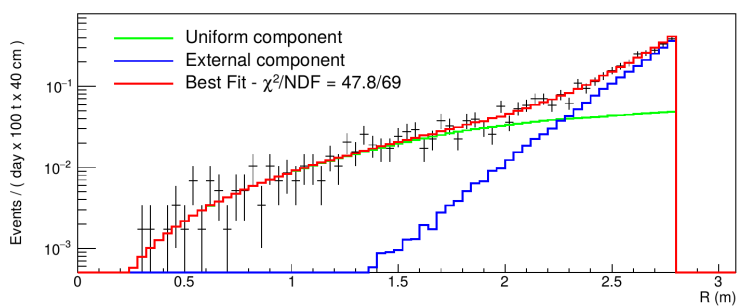

(d) Radial distribution.

Figure 4: Distributions and fits of the components of the multivariate fit. 
the $\boldsymbol{T F C}$-tagged and $\boldsymbol{T F C}$-subtracted energy spectra mentioned in Sec. 3.1.1, the radial distribution, and the so-called pulse shape variable distribution, a parameter tuned to distinguish electron and positron events. It is used to disentange the ${ }^{11} \mathrm{C} e^{+}$decays originating from the remaining ${ }^{11} \mathrm{C}$ in the $T F C$-subtracted spectrum. Examples of the distributions and fits are shown in Fig. 4.

\subsection{High energy region analysis}

The HER itself is divided into two subregions, HER-I (3.2 - 5.7 MeV) and HER-II $(5.7-16 \mathrm{MeV})$. The main backgrounds in HER-I and HER-II are natural radioactivity from ${ }^{208} \mathrm{Tl}$, and external gamma rays following neutron capture processes on the SSS, respectively. The value of $5.7 \mathrm{MeV}$ has been chosen this way because the ${ }^{208} \mathrm{Tl}$ decays never exceed $5 \mathrm{MeV}$.

\subsubsection{Data selection}

The HER analysis uses the following selection cuts:

- Muon cut: same as in the LER analysis (Sec. 3.1.1).

- Neutron cut: removes cosmogenic neutron captures on ${ }^{14} \mathrm{C}$ by applying a $2 \mathrm{~ms}$ veto after all muons.

- Fast cosmogenics cut: removes cosmogenic isotopes by applying a $6 \mathrm{~s}$ veto after each internal muon.

- Run start/break cut: remove fast cosmogenic isotopes from potential muons missed during breaks in data taking.

- ${ }^{10} \mathrm{C}$ cut: remove cosmogenic ${ }^{10} \mathrm{C}$ by vetoing a spherical volume of radius $0.8 \mathrm{~m}$ around all muon-induced neutron captures.

- ${ }^{214} \mathbf{B i}^{2}{ }^{214}$ Po cut: removes coincidence pairs of ${ }^{214} \mathrm{Bi}$ and ${ }^{214} \mathrm{Po}$, daughters of the radioactive decay of ${ }^{222} \mathrm{Rn}$. This cut is also used in the LER analysis and its efficiency is $\sim 92 \%$.

- Fiducial volume cut: $z<2.5 \mathrm{~m}$ to remove the events in the top layer of the LS; applied only in HER-I.

\subsubsection{Radial fit}

The most important feature of the HER analysis is that independent fits are performed only on the radial distributions of the selected events. This means that there is no assumption on the neutrino energy spectrum, which allows us to probe for deviations from the MSW theory. Examples of the fits are shown in Fig. 5. The rate of ${ }^{8} \mathrm{~B}$ neutrinos is extracted from these fits, while the rate of hep neutrinos is obtained via a simple counting analysis for energies above $10 \mathrm{MeV}$.

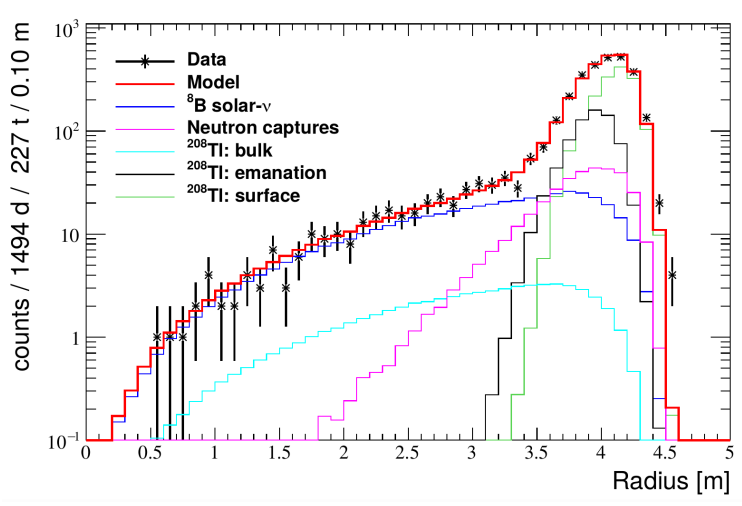

(a) HER-I

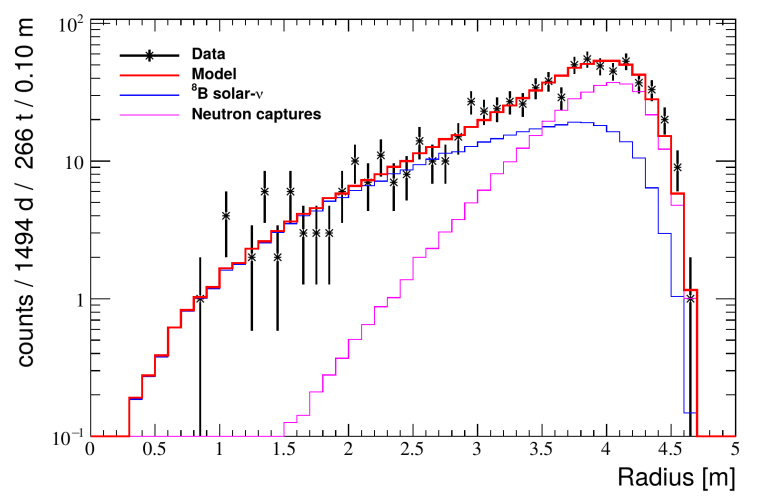

(b) HER-II

Figure 5: Examples of the data sample (black stars), fit results (red) and reference shapes (see legends) of the HER-I and HER-II analyses. 


\section{Results and implications}

The LER and HER analyses result in a comprehensive study of all solar neutrino types [5]:

- $p p$ : 9.5\% precision (improved from $11.5 \%$ );

- pep: $16 \%$ precision (improved from $21.5 \%$ ), discovery for the first time ( $>5 \sigma$ measurement);

- ${ }^{7} \mathrm{Be}: 2.7 \%$ precision (improved from $4.5 \%$ ), two times more precise than the theoretical prediction;

- ${ }^{8} \mathrm{~B}: 8 \%$ (improved from $18.5 \%$ );

- hep: $90 \%$ CL upper limit (for the first time);

- CNO: 95\% CL upper limit;

\subsection{Solar models}

Using the LER results on $p p$ and ${ }^{7} \mathrm{Be}$ neutrinos, one can calculate the relative intensity of the $p p-I$ and $p p-I I$ terminations of the $p p$-chain depicted in Fig. 1:

$$
R_{\mathrm{I} / \mathrm{II}}=\frac{2 \phi\left({ }^{7} \mathrm{Be}\right)}{\phi(p p)-\phi\left({ }^{7} \mathrm{Be}\right)},
$$

where $\phi$ denotes the neutrino flux of the given type. The theoretical predictions are $R_{\mathrm{I} / \mathrm{II}}=0.180 \pm 0.011$ and $R_{\mathrm{I} / I \mathrm{I}}=0.161 \pm 0.010$ for HM- and LM-SSM, respectively. The experimental result is $R_{\mathrm{I} / \mathrm{II}}=0.178 \pm 0.027$ which is compatible with the expected values.

Another way to take a look at solar metallicity is to use only the results on ${ }^{7} \mathrm{Be}$ and ${ }^{8} \mathrm{~B}$ neutrino fluxes which are most dependent on the HM- and LM-SSM predictions. As can be seen from Fig. 6a, the Borexino measurement gives a weak hint towards HM-SSM. In a global fit together with all solar experiments and KamLAND, the hint further weakens. In this fit, the oscillation parameters $\theta_{12}$ and $\Delta m_{12}^{2}$ are left free to vary, and the results returned by the fit are consitent with the ones obtained in [2].

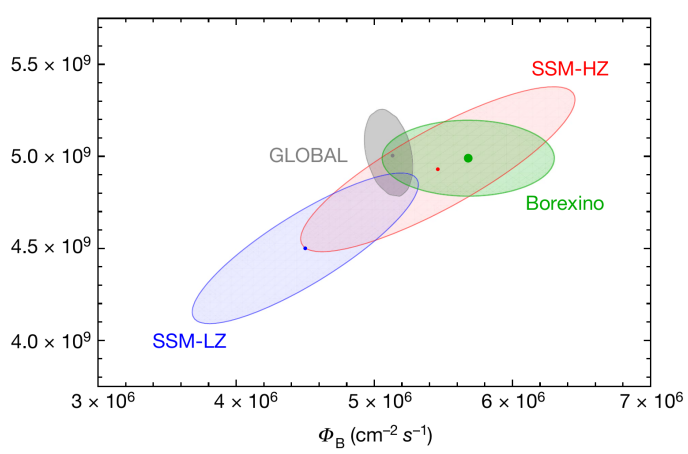

(a)

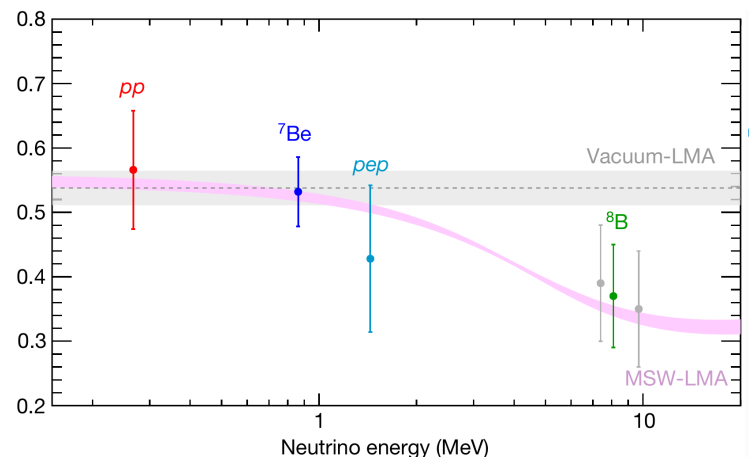

(b)

Figure 6: (a) Allowed $1 \sigma$ theoretical regions of the ${ }^{8} \mathrm{~B}$ and ${ }^{7} \mathrm{Be}$ fluxes according to LM-SSM (blue) and HM-SSM (red), compared to the Borexino measurement (green) and a global fit (grey). (b) Electron survival probability values in different energy regions using the Borexino measurement compared to the vacuum oscillation model and the MSW-LMA model.

\subsection{Neutrino oscillations}

In Fig. $6 \mathrm{~b}$ one can see the Borexino results on the electron survival probability based on different $p p$-chain neutrino fluxes. The Borexino results are represented by points, while the theoretical predictions according to the vacuum oscillation model and the MSW-LMA modal are represented by the grey and the pink bands, respectively. Borexino is the only experiment that can probe $P_{e e}$ in both vacuum and matter dominated regions. The results disfavour vacuum oscillations at $95 \% \mathrm{CL}$. 


\subsection{Thermodynamic equilibrium of the Sun}

Using the neutrino rates and the nuclear reactions from Fig. 1, one can calculate the total power to be $L=$ $(3.89 \pm 0.42) \times 10^{33} \mathrm{erg} \mathrm{s}^{-1}$ which is compatible the photon output $L=(3.846 \pm 0.015) \times 10^{33} \mathrm{erg} \mathrm{s}^{-1}$. This finding is an experimental confirmation of the nuclear origin of the solar power. Since Borexino gives a real-time picture of the core Sun, the finding also proves the thermodynamic equilibrium of the Sun over the time scale of $10^{5}$ years. Moreover, this latest Borexino result holds the best precision obtained by a single solar neutrino experiment.

\section{Outlook}

The CNO neutrino analysis is going to be carried out on the Borexino Phase-III data which starts from July 2016. The main challenge of this measurement is the extreme similarity of the $\mathrm{CNO}$ spectral shape to that of ${ }^{210} \mathrm{Bi}$ and pep neutrinos which can be seen in Fig. 7a. One of the strategies of the CNO analysis is to constrain the rate of ${ }^{210} \mathrm{Bi}$, a $\beta$-emitter, by measuring the events coming from its daughter, ${ }^{210} \mathrm{Po}$. It is the only $\alpha$ emitter, and can be measured on an event-by-event basis using pulse shape information. An important part of this estimation is to disentangle the non-equilibrium vessel contamination of ${ }^{210} \mathrm{Po}$. This is made possible due to the detector thermal insulation campaign conducted in 2015. The resulting thermal stabilization can be seen in Fig. $7 b$, where the ${ }^{210} \mathrm{Po}$ rate is measured in cubic volumes uniformly dividing the inner vessel. Sensitivity studies with toy Monte Carlo show possibility of CNO measurement between $3 \sigma$ and $4 \sigma$.

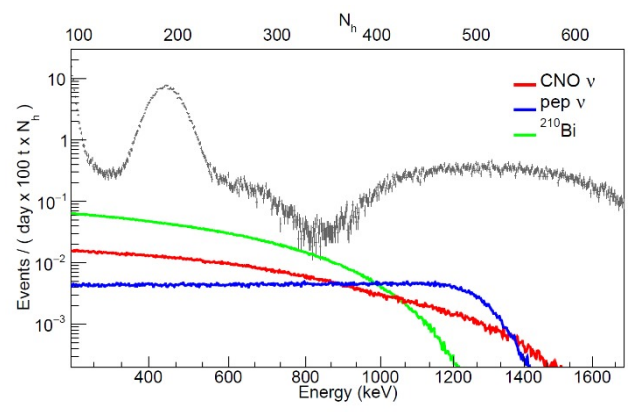

(a)

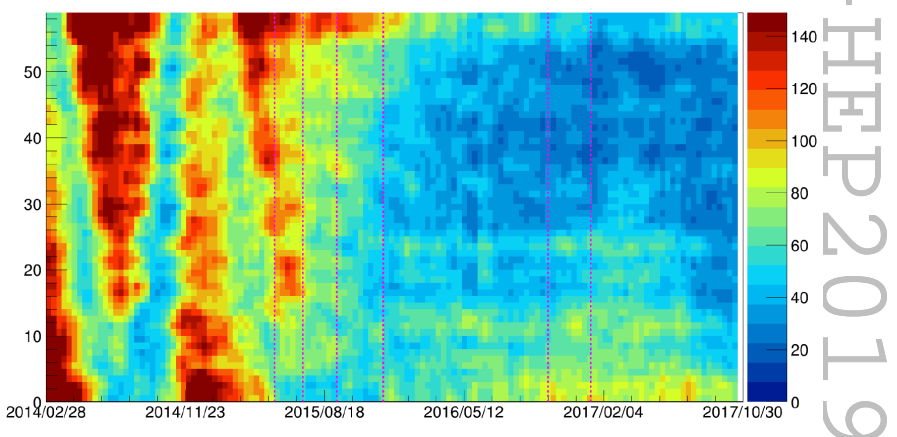

(b)

Figure 7: (a) The Borexino neutrino data (black) and the theoretical spectra of ${ }^{210} \mathrm{Bi}$ (green), pep (blue), and CNO (red) neutrinos. (b) The dependence of ${ }^{210} \mathrm{Po}$ rate in cubic volumes on time.

\section{References}

[1] N. Vinyoles, A. M. Serenelli, F. L. Villante, S. Basu, J. Bergström, M. C. Gonzalez-Garcia, M. Maltoni, C. Peña-Garay, and N. Song, "A new Generation of Standard Solar Models," Astrophys. J., vol. 835, no. 2, p. 202, 2017.

[2] I. Esteban, M. C. Gonzalez-Garcia, M. Maltoni, I. Martinez-Soler, and T. Schwetz, "Updated fit to three neutrino mixing: exploring the accelerator-reactor complementarity," JHEP, vol. 01, p. 087, 2017.

[3] G. Bellini et al., "Final results of Borexino Phase-I on low energy solar neutrino spectroscopy," Phys. Rev., vol. D89, no. 11, p. $112007,2014$.

[4] G. Alimonti et al., "The Borexino detector at the Laboratori Nazionali del Gran Sasso," Nucl. Instrum. Meth., vol. A600, pp. 568-593, 2009.

[5] M. Agostini et al., "Comprehensive measurement of pp-chain solar neutrinos,” Nature, vol. 562, p. $505,2018$. 